EPJ Web of Conferences 37, 09012 (2012)

DOI: $10.1051 /$ epjconf/20123709012

(C) Owned by the authors, published by EDP Sciences, 2012

\title{
Meson Screening Masses in Thermal QCD
}

\section{Piotr Czerski ${ }^{\mathrm{a}}$}

The H. Niewodniczański Institute of Nuclear Physics, Polish Academy of Sciences, ul. Radzikowskiego 152, 31-342 Kraków, Poland

\begin{abstract}
The quark-gluon plasma (QGP) excitations, corresponding to the scalar and pseudoscalar meson quantum numbers, for different temperatures are calculated. Analysis is performed in the Hard Thermal Loop (HTL) Approximation and leads to a better understanding of the excitations of QGP in the deconfined phase and is also of relevance for lattice studies.
\end{abstract}

\section{Spatial correlation function in HTL approximation}

The spatial correlations are exponentially suppressed at large values of $z$

$$
\mathcal{G}(z) \underset{z \rightarrow+\infty}{\sim} e^{-m_{s c r} z}
$$

and the suppression parameter, which is governing the large distance behavior of the correlator is the screening mass $m_{s c r}$. It gives information on the nature of the excitations characterizing the QGP phase.

One can express the spatial correlation function through the finite momentum spectral function of the quark-antiquark excitations in QGP which in the scalar channel is represented by $\sigma_{S}(\omega, \boldsymbol{p})$.

$$
\mathcal{G}_{S}(z)=\int_{-\infty}^{+\infty} \frac{d p_{z}}{2 \pi} e^{i p_{z} z} \int_{-\infty}^{+\infty} d \omega \frac{\sigma_{S}\left(\omega, \boldsymbol{p}_{\perp}=0, p_{z}\right)}{\omega} .
$$

Scalar meson correlation function in HTL approximation is calculated in the way similar to the pseudoscalar one [1].

$$
\begin{aligned}
\sigma_{S}(\omega, \boldsymbol{p}) & =N_{f} N_{c} \int \frac{d^{3} k}{(2 \pi)^{3}}\left(e^{\beta \omega}-1\right) \int_{-\infty}^{+\infty} d \omega_{1} \int_{-\infty}^{+\infty} d \omega_{2} \tilde{n}\left(\omega_{1}\right) \tilde{n}\left(\omega_{2}\right) \delta\left(\omega-\omega_{1}-\omega_{2}\right) \times \\
& \times\left\{(1+\hat{\boldsymbol{k}} \cdot \hat{\boldsymbol{q}})\left[\rho_{+}\left(\omega_{1}, k\right) \rho_{+}\left(\omega_{2}, q\right)+\rho_{-}\left(\omega_{1}, k\right) \rho_{-}\left(\omega_{2}, q\right)\right]+\right. \\
& \left.+(1-\hat{\boldsymbol{k}} \cdot \hat{\boldsymbol{q}})\left[\rho_{+}\left(\omega_{1}, k\right) \rho_{-}\left(\omega_{2}, q\right)+\rho_{-}\left(\omega_{1}, k\right) \rho_{+}\left(\omega_{2}, q\right)\right]\right\},
\end{aligned}
$$

with

$$
\rho_{ \pm}(\omega, k)=\frac{\omega^{2}-k^{2}}{2 m_{q}^{2}}\left[\delta\left(\omega-\omega_{ \pm}\right)+\delta\left(\omega+\omega_{\mp}\right)\right]+\beta_{ \pm}(\omega, k) \theta\left(k^{2}-\omega^{2}\right)
$$

and

$$
\beta_{ \pm}(\omega, k)=-\frac{m_{q}^{2}}{2} \frac{ \pm \omega-k}{\left[k(-\omega \pm k)+m_{q}^{2}\left( \pm 1-\frac{ \pm \omega-k}{2 k} \ln \frac{k+\omega}{k-\omega}\right)\right]^{2}+\left[\frac{\pi}{2} m_{q}^{2} \frac{ \pm \omega-k}{k}\right]^{2}},
$$

\footnotetext{
${ }^{a}$ e-mail: piotr.czerski@ifj.edu.pl
}

This is an Open Access article distributed under the terms of the Creative Commons Attribution License 2.0, which permits unrestricted use, distribution, and reproduction in any medium, provided the original work is properly cited. 
Table 1. The pseudo-scalar and scalar screening mass of the interacting QGP.

\begin{tabular}{cccc}
\hline$T / T_{c}$ & $m_{s c r}^{\text {free }} / 2 \pi T$ & $m_{P S}^{\text {scr }} / 2 \pi T$ & $m_{S}^{\text {scr }} / 2 \pi T$ \\
\hline 1 & 1.1007 & 0.7581 & 0.70402 \\
2 & 1.0603 & 0.8136 & 0.73859 \\
4 & 1.0441 & 0.8950 & 0.84691 \\
10 & 1.0331 & 1.0192 & 0.99892 \\
\hline
\end{tabular}

where $\omega_{\mp}(k)$ are dispersion relations for plasmino and quasi-particle excitations. For high energy $\omega \rightarrow \infty$ the spectral function $\sigma_{S}\left(\omega, p_{z}\right) \rightarrow \omega^{2}$ and (1) diverge.

To get a finite results for $\mathcal{G}(z)$ one has to regularize integrals. For the non interacting QGP the problem was solved for all mesonic channels in [2] adopting adopting Pauli-Villars regularization scheme. In the interacting case the problem is much more complicated since the meson spectral function is obtained only numerically and one has to adopt a numerical regularization.

For numerical regularization of the integrals we follow the method proposed in [3]. We calculate numerically the difference of two functions which is converging to zero for high energies and allows to perform all integrations numerically for a given $p_{z}$ momentum. $\sigma_{S}^{\text {diff }}\left(\omega, p_{z}\right)=\sigma_{S}^{\text {free }}\left(\omega, p_{z}\right)-\sigma_{S}\left(\omega, p_{z}\right)$, with the asymptotic mass $m=\sqrt{2} m_{q}$ used in the free case. The final result is: $\mathcal{G}^{\text {full }}(z)=\mathcal{G}^{\text {free }}(z)-$ $\mathcal{G}^{\text {diff }}(z)$. For large $z$ the final results is fitted to the $\mathcal{G}^{f u l l}(z)=\sum_{i=1}^{n} b_{i} \frac{e^{-m_{i} z}}{z}$. The screening mass $m_{s c r}$ is a smallest between all $m_{i}$ masses for a given $\mathrm{T}$.

\section{Results}

In Fig 1 we show the temperature dependence of the screening masses with comparison with the recent lattice results. In Table 1 we summarize screening masses for all temperatures and channels, where $m_{s c r}^{\text {free }}=2 \sqrt{\pi^{2} T^{2}+m^{2}}$ is the free screening mass, $m=\sqrt{2} m_{q}$ is the asymptotic mass and $m_{q}=g(T) T / \sqrt{6}$ is the thermal gap mass of the quark. $m_{P S}^{s c r}$ and $m_{S}^{s c r}$ are the pseudo-scalar and scalar screening mass of the interacting QGP.

The numbers in the Table 1 are calculated assuming $N_{f}=2$ and the transition temperature, following [6], $T_{c}=202 \mathrm{MeV}$. The ratio $T_{c} / \Lambda_{\overline{M S}}=0.7721$ for the $N_{f}=2$ case is taken from [7]. The running of the gauge coupling is given by the two-loop perturbative beta-function, leading to the expression:

$$
g^{-2}(T)=2 b_{0} \log \frac{\mu}{\Lambda_{\overline{M S}}}+\frac{b_{1}}{b_{0}} \log \left\{2 \log \frac{\mu}{\Lambda_{\overline{M S}}}\right\},
$$

where $b_{0}=\frac{1}{16 \pi^{2}}\left(11-2 \frac{N_{f}}{3}\right), b_{1}=\frac{1}{\left(16 \pi^{2}\right)^{2}}\left(102-38 \frac{N_{f}}{3}\right)$. We adopt $\mu=1.142 \pi T$ suggested in [6]. Finally the thermal gap mass of the quark $m_{q}=g(T) T / \sqrt{6}$.

\section{Conclusions}

The main goal of this work is to calculate the asymptotic mass of the scalar and pseudo scalar meson in the interacting quark gluon plasma for different temperatures.

The meson screening masses in the scalar and pseudoscalar channels are calculated from the momentum dependent meson spectral functions in the Hard Thermal Loop Approximation (HTL) of the QCD $[1,3]$. A new subtraction procedure is invented to get an UV finite result. It is a numerical approach, which requires the analytical form of the mesonic spatial correlation functions in the free case [2] in order to calculate the divergent integrals. Results are compared to the recent QCD lattice results $[4,5]$.

This analysis leads to a better understanding of the excitations of Quark Gluon Plasma in finite temperatures and is of relevance for interpreting lattice results. 

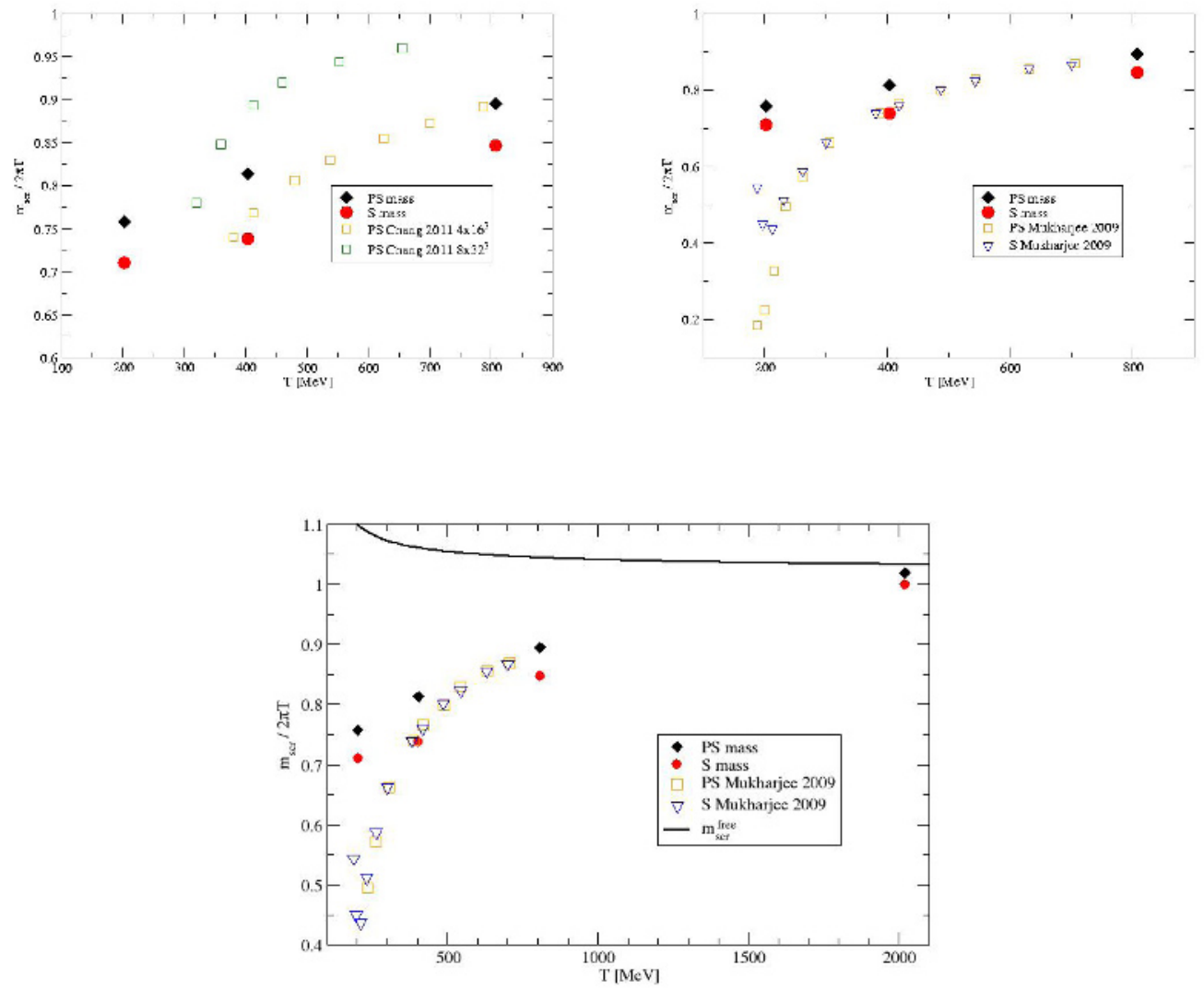

Fig. 1. Temperature dependence of the scalar and pseudoscalar screening masses compared with the lattice results extracted from Ref. [4] (upper left Fig.) and from Ref. [5] (upper right Fig.). Lower Fig. shows the temperature dependence of the scalar and pseudoscalar screening masses compared with the lattice results extracted from Ref. [5] and compared to the $m_{s c r}^{\text {free }}$.

\section{References}

1. W.M. Alberico, A. Beraudo, P. Czerski and A. Molinari, Nucl. Phys. A775 (2006) 188

2. P. Czerski, Cent. Eur. J. Phys. 10 (2012) 342

3. W.M. Alberico, A. Beraudo, A.Czerska, P. Czerski and A. Molinari, Nucl. Phys. A 792152 (2007)

4. M. Cheng et al., Eur. Phys. J. C71 (2011) 1564

5. S. Mukherjee, Nucl. Phys. A820 (2009) 283C

6. O. Kaczmarek and F. Zantow, Phys. Rev. D71 (2005) 114510

7. F. Karsch, E. Laermann, and A. Peikert, Phys. Lett. B478 (2000) 447 